\title{
Predictores de la percepción de la enfermedad mental entre pacientes psicóticos y no psicóticos
}

\author{
Andrea Lang Dr \\ Gerlinde Berghofer Dr \\ Anita Kager M.A. \\ Egbert Steiner \\ Friedrich Schmidl Dr \\ Stephan Rudas MD
}

Servicios comunitarios de Salud Mental Steigenteschgasse 142 A-1220 Vienna AUSTRIA

\begin{abstract}
RESUMEN - Los objetivos de este estudio eran $1^{\circ}$ ) elaborar un índice para una mejor percepción de la enfermedad mental y $2^{\circ}$ ) examinar los predictores para lograr dicha percepción dentro de una muestra regionalmente representativa de 418 pacientes.
\end{abstract}

La percepción se evaluó por medio de un índice basado en tres preguntas. De forma adicional a las preguntas,la estimación del entrevistador sobre la percepción de los pacientes también se tuvo en cuenta para el índice. Una buena percepción y una percepción moderada se asociaron con edad temprana, estado civil casado/cohabitando, existencia de una relación de confianza íntima, diagnóstico de trastorno de estado de ánimo, ansiedad o trastorno de adaptación y dependencias del alcohol y/o de drogas, levedad de la enfermedad, salud física y mental autovalorada como mala, edad temprana en el primer tratamiento,alta valoración del tratamiento,y pocos ingresos hospitalarios durante el periodo de estudio. Por medio de un análisis discriminante se alcanzó una clasificación correcta de grupo en el $44 \%$ del número total de pacientes.

Se halló que el índice suponía una herramienta rápida y aceptable que podía encontrar aplicación como método de ponderación de estos niveles de percepción de la enfermedad mental.

\section{Introducción}

Hasta el comienzo de los años ochenta los expertos asumían que la percepción de la enfermedad era una variable dicotómica que seguía el 'concepto de todo o nada' (Van Put- ten et al. 1976, Lin et al. 1979, Heinrichs et al. 1985). Investigaciones posteriores detectaron que la percepción abarcaba una variedad de fenómenos basados en diversas magnitudes (Wciórka 1988, Greenfield et al. 1989; David et al. 1990, Amador et al. 1991, 1993). 
Por ello, Amador \& Strauss (1993) desarrollaron una Escala para Evaluar el Desconocimiento de los Trastornos Mentales (Scale to Assess Unawareness of Mental Disorders SUMD). La escala SUMD incluye las magnitudes: conocimiento de la enfermedad, necesidad de tratamiento médico, conocimiento de las consecuencias sociales del trastorno mental y conocimiento y adscripción de indicios específicos.

Los resultados de los estudios sobre percepción de la enfermedad mental no pueden compararse entre ellos, debido al gran número de instrumentos heterogéneos basados en diferentes conceptualizaciones. Algunos estudios mostraron como el desarrollo social y la percepción están asociados de forma positiva (Soskis y Bowers 1969, Lysker y Bell 1994, Peralta y Cuesta 1994). McGlashan y Carpenter 1981 demostraron que la percepción se asocia con la dimensión de la estructura social. Fennig et al. (1996) hallaron que la percepción se asocia con el estado civil casado, y que los pacientes esquizofrénicos muestran una menor percepción que las personas con trastornos bipolares, depresiones graves u otros trastornos psicóticos. Carpenter et al. (1973) demostraron que el $81 \%$ del total de los pacientes esquizofrénicos carecen de tal percepción. Por el contrario, David et al. (1992) pusieron de relieve que no sólo los pacientes esquizofrénicos presentan un escaso grado de percepción. Amador et al. (1994) también mostraron como las puntuaciones sobre percepción de la enfermedad mental de los individuos con trastornos bipolares son más bajas en comparación con otros grupos diagnósticos. En la bibliografía, la relación entre la percepción y las impresiones clínicas generales permanece poco clara. Los estudios revelan resultados dispares e inconsistentes. Amador et al. (1991), por ejemplo, sostenía que los estudios más detallados nunca lleva- ban a cabo el examen de la asociación entre psicopatología y nivel de percepción (véase Bartko et al. 1988, McEvoy et al. 1989a, 1993). Posteriormente, Amador et al. (1994) aportaron información acerca de que la percepción presenta correlación de una forma significativa y positiva, con la gravedad de la psicopatología, sin embargo, dicha correlación no era significativa cuando el diagnóstico se reevaluaba a fondo. David et al. (1992) observaron que los valores totales de la percepción estaban inversamente relacionados, de forma moderada, con el índice general de psicopatología.

La cumplimiento de la medicación no se vio que estuviese asociada de forma positiva con la percepción en los pacientes esquizofrénicos (Lin et al. 1979, Bartko et al. 1988, McEvoy et al. 1989a , 1989b, Lysaker y Bell 1994), mientras que, entre los pacientes hospitalizados McEvoy et al. (1989b) no encontraron que el cumplimiento de la medicación fuera un buen predictor de la percepción. McEvoy señaló una moderada correlación inversa entre el incumplimiento de la medicación y la percepción. Considerando estos resultados, los autores investigaron también una asociación positiva con la percepción (Lin et al. 1979, McGlashan y Carpenter 1981, Heinrichs et al. 1985, Bartko et al. 1988, McEvaoy et al. 1989b), sin embargo, algunas investigaciones anteriores no pudieron confirmar este hallazgo (Eskey 1958, Van Putten et al. 1976). Los pacientes con afecciones de corta duración y bajas puntuaciones en relación con la percepción presentaban un alto riesgo de ser hospitalizados de nuevo (McEvoy et al. 1993). La percepción no predecía el número de hospitalizaciones (Lysaker y Bell 1994).

El principal objetivo de la presente investigación es ensayar un índice de percepción en una muestra de pacientes psicóticos y no psicóticos. El término percepción se utilizó de forma descriptiva para denotar el fenómeno 
por el cual los pacientes psiquiátricos eran capaces de reconocer que estaban enfermos, que conocían su enfermedad o que eran capaces de describir los síntomas que constituían su diagnóstico y/o si tenían expectativas de tratamiento. Los factores asociados con la percepción de la enfermedad mental se identificarían a través de la selección de una muestra de un paciente interno y de un paciente externo.

\section{Métodos}

\section{Sujetos}

Hace más de veinte años, durante el proceso de la reforma psiquiátrica, se estableció en Viena, Austria, un sistema público de salud mental global, por sectores y coordinado (Rudas 1990). En Abril de 1980 se abrió el primero de ocho centros de salud mental regional. Cada una de las ocho zonas de influencia tenía una población de $147.000-235.000$ personas.

Los sujetos eran pacientes externos en cuatro de estos centros públicos vieneses de salud mental, así como pacientes internos de dos establecimientos psiquiátricos (uno en un hospital psiquiátrico y el otro en el ala de Psiquiatría de un hospital general). La inclusión de los pacientes comenzó en Enero de 1995 y finalizó en Abril de 1996. Los criterios de exclusión eran: que no desearan participar, psicosis en fase activa, demencia, debilidad mental y conocimientos insuficientes de alemán. La muestra se divide en pacientes de primer contacto (en relación con la institución) y pacientes de larga duración (lo que significa que la duración del tratamiento de un paciente externo de larga duración era de más de 3 meses, respectivamente; los pacientes internos de larga dura- ción presentaban al menos dos hospitalizaciones). La totalidad de la muestra la integraban 448 pacientes. De ellos, 23 no se incluyeron porque no completaron la primera entrevista. No se encontraron diferencias significativas entre los pacientes excluidos e incluidos en términos de características demográficas y clínicas. La población del estudio final la integraban 425 pacientes. Todos ellos otorgaron consentimiento por escrito para someterse a una entrevista. En la línea base 165 (39\%) estaban hospitalizados. $202(48 \%)$ eran pacientes de primer contacto, y $223(52 \%)$ eran pacientes de larga duración. La inclusión de éstos últimos se realizó de forma aleatoria en 6 días clave. Se pidió a los pacientes de primer contacto que su participación tuviera lugar en la secuencia en que fueron apareciendo durante el periodo de inclusión del estudio.

\section{Instrumentación y recogida de información:}

Se realizó una entrevista semi-normalizada para registrar las características demográficas y la información clínica y de tratamiento. Tanto la situación clínica como la habilidad de los pacientes para afrontar situaciones fueron evaluadas con el Global Assessment of Functioning Scale (GAF) utilizando una revisión de GAS (Endicott et al. 1976) y de CGAS (Shaffer et al. 1978). La gravedad que en aquel momento presentaba la enfermedad fue evaluada con la Clinical Global Impres sions Scale (CGI) (Instituto Nacional de Salud Mental 1976). Se puntuó la salud mental y física presente y futura sobre una escala analógica visual de $100 \mathrm{~mm}$.

El pronóstico se consideraba 'optimista' cuando la diferencia entre la salud esperada y la real en aquel momento, era de al menos 10 puntos. El pronóstico se consideraba 'pesi- 
mista' cuando la diferencia era inferior a 10 puntos,cero o negativa. Fue evaluado por personal clínico y autovalorado por los pacientes.

Para este estudio, se desarrolló un nuevo índice para la medición de la percepción de la enfermedad mental -basado en cuatro apartados-. Cada uno de los apartados siguientes se valoraba con un punto: si el paciente reconocía encontrarse enfermo, si el paciente era capaz de nombrar el diagnóstico y/o los síntomas correctos, si el paciente tenía al menos una expectativa de tratamiento. Teniendo en cuenta las expectativas de tratamiento, se preguntaba a los pacientes si consideraban que los síntomas, la situación laboral, el entorno de residencia, las relaciones sociales, las tareas cotidianas y la estabilidad en el estado de salud, mejorarían a través del tratamiento. De forma adicional, se realizaba una clasificación de la importancia de las expectativas de tratamiento y se valoraban con un punto, únicamente cuando el paciente entrevistado valoraba una de dichas expectativas de tratamiento como importante o bastante importante. Además, se añadía un punto cuando el entrevistador valoraba al paciente como perceptivo. Las puntuaciones de los cuatro apartados se sumaban para obtener una puntuación global de la percepción de la enfermedad. De acuerdo con la suma de puntuaciones, los cuatro posibles niveles de percepción eran: 'buena percepción' (4 puntos), 'percepción moderada' (3 puntos), 'escasa percepción' (2 puntos) y 'muy escasa percepción' (1 ó 0 puntos).

Los entrevistadores eran psicólogos con experiencia suficiente en trabajo con pacientes psiquiátricos. Los entrevistadores eran independientes del tratamiento del paciente. Antes del estudio, los entrevistadores habían superado con éxito la calibración inicial de sus criterios de clasificación. Los procesos estadísticos se llevaron a cabo utilizando Sta tistical Package for Social Sciences (SPSS)
6.1.3. La información se analizó utilizando el test ANOVA (análisis de variaciones) sobre una sola variable y el test $\mathrm{c}^{2}$. Se realizó un análisis discriminante para determinar las características relacionadas con la percepción de la enfermedad del paciente.

\section{Resultados}

\section{Detalles demográficos}

De entre el total de la muestra del estudio, siete pacientes fueron clasificados como 'no enfermos' en la Escala CGI. Por lo tanto, de la muestra total $(\mathrm{N}=425)$ se disponía de información sobre 418 personas para la evaluación de la percepción. Las características demográficas de la muestra se presentan en la tabla I.

El 53\% de los sujetos del estudio eran hombres. Los pacientes fueron diagnosticados por personal clínico de acuerdo con los criterios de diagnóstico del DSM IV. Cerca de la mitad de los pacientes sufrían esquizofrenia $(40 \%)$. El $20 \%$ eran pacientes no psicóticos, es decir, padecían ansiedad o trastornos de adaptación y dependencias del alcohol y las drogas. La muestra presentaba una edad media de 44,0 años (SD = 15,2). El $40 \%$ eran solteros. Más de un tercio de todos los pacientes (34\%) no había completado una educación superior y el $78 \%$ estaba desempleado. La duración de la enfermedad en el $37 \%$ de los casos era de dos años y en el $31 \%$ era de más de 10 años. El 34\% nunca había sido hospitalizado, el $16 \%$ presentaba un solo ingreso previo, el $29 \%$ entre dos y cinco ingresos y el $21 \%$ más de seis ingresos. La mayoría de los pacientes (65\%) mostraron una percepción moderada o una buena percepción. Veinte pacientes (12\%) de entre la muestra de no psicóticos $(\mathrm{n}=$ 161) mostraron 'muy escasa percepción'. 
Tabla I

Aspectos demográficos $(\mathrm{N}=418)$

\begin{tabular}{|c|c|c|}
\hline Características & $\mathrm{n}$ & $\%$ \\
\hline \multicolumn{3}{|l|}{ Edad } \\
\hline Media (años) & 44,0 & $15,2(\mathrm{SD})$ \\
\hline \multicolumn{3}{|l|}{ Sexo } \\
\hline Hombre & 222 & 53 \\
\hline Mujer & 196 & 47 \\
\hline \multicolumn{3}{|l|}{ Estado civil } \\
\hline Soltero & 167 & 40 \\
\hline Casado/cohabitando & 109 & 26 \\
\hline Divorciado/separado & 117 & 28 \\
\hline Viudo & 25 & 6 \\
\hline \multicolumn{3}{|l|}{ Empleo } \\
\hline Sí & 92 & 22 \\
\hline No & 325 & 78 \\
\hline \multicolumn{3}{|l|}{ Educación profesional } \\
\hline Terminada & 276 & 66 \\
\hline No terminada & 142 & 34 \\
\hline \multicolumn{3}{|l|}{ Diagnóstico (DSM IV) } \\
\hline Trastornos orgánicos & 19 & 5 \\
\hline Dependencia alcohol/drogas & 80 & 19 \\
\hline Trastornos esquizofrénicos & 168 & 40 \\
\hline Trastornos del humor & 53 & 13 \\
\hline Trastornos de ansiedad/adaptación & 62 & 15 \\
\hline Trastornos de personalidad & 34 & 8 \\
\hline \multicolumn{3}{|l|}{ Admisiones previas } \\
\hline 0 & 140 & 34 \\
\hline 1 & 68 & 16 \\
\hline $2-5$ & 122 & 29 \\
\hline 6 y más & 88 & 21 \\
\hline \multicolumn{3}{|l|}{ Duración de enfermedad } \\
\hline Media (años) & 8,8 & $10,9(\mathrm{SD})$ \\
\hline \multicolumn{3}{|l|}{ Percepción } \\
\hline Muy pobre & 67 & 16 \\
\hline Pobre & 81 & 19 \\
\hline Moderada & 133 & 32 \\
\hline Buena & 137 & 33 \\
\hline \multicolumn{3}{|l|}{ Percepción de pacientes no psicóticos } \\
\hline Muy pobre & 20 & 12 \\
\hline Pobre & 24 & 15 \\
\hline Moderada & 66 & 41 \\
\hline Buena & 51 & 32 \\
\hline
\end{tabular}

Características demográficas, factores relacionados con historial médico y tratamiento y niveles de percepción

La distribución de las características demográficas y de historial en los cuatro niveles de percepción (= índice) se presenta en las tablas II y III.

Los resultados mostraron que no se da una asociación entre la percepción y el sexo del sujeto (tabla II). Los pacientes más jóvenes, durante el periodo del estudio y cuando recibieron atención psiquiátrica por primera 
Tabla II

Asociación entre percepción y características demográficas y anamnésicas

\begin{tabular}{|c|c|c|c|c|c|c|c|}
\hline & $\begin{array}{l}\text { Muy pobre } \\
\text { "insight" }\end{array}$ & $\begin{array}{l}\text { Pobre } \\
\text { "insight" }\end{array}$ & $\begin{array}{l}\text { Moderado } \\
\text { "insight" }\end{array}$ & $\begin{array}{l}\text { Bueno } \\
\text { "insight" }\end{array}$ & & & \\
\hline Variable & $\begin{array}{c}\mathrm{n}=67 \\
\%\end{array}$ & $\begin{array}{c}\mathrm{n}=81 \\
\%\end{array}$ & $\begin{array}{c}\mathrm{n}=133 \\
\%\end{array}$ & $\begin{array}{c}\mathrm{n}=137 \\
\%\end{array}$ & $\chi^{2}$-Test & df & $\begin{array}{c}\text { sign. } \\
\text { valor } \mathrm{P}^{*}\end{array}$ \\
\hline \multicolumn{8}{|l|}{ Sexo } \\
\hline Hombre & 55 & 48 & 59 & 49 & \multirow{2}{*}{$\chi^{2}=40$} & \multirow[t]{2}{*}{3} & \multirow[t]{2}{*}{ n.s. } \\
\hline Mujer & 45 & 52 & 41 & 51 & & & \\
\hline \multicolumn{8}{|l|}{ Estado civil } \\
\hline Soltero & 35 & 45 & 41 & 39 & \multirow[t]{4}{*}{$\chi^{2}=35.1$} & \multirow[t]{4}{*}{9} & \multirow[t]{4}{*}{$<.001$} \\
\hline Casado/cohabitando & 12 & 25 & 13 & 28 & & & \\
\hline Divorciado/separado & 33 & 26 & 24 & 29 & & & \\
\hline Viudez & 20 & 4 & 2 & 4 & & & \\
\hline \multicolumn{8}{|l|}{ Confidente íntimo } \\
\hline Sí & 73 & 77 & 86 & 88 & \multirow{2}{*}{$\chi^{2}=9.5$} & \multirow[t]{2}{*}{3} & \multirow[t]{2}{*}{.023} \\
\hline No & 27 & 23 & 14 & 12 & & & \\
\hline \multicolumn{8}{|l|}{ Diagnóstico (DSM) } \\
\hline Trastornos orgánicos & 9 & 7 & 3 & 2 & \multirow[t]{6}{*}{$\chi^{2}=33.7$} & \multirow[t]{6}{*}{15} & \multirow[t]{6}{*}{.004} \\
\hline Dependencia alcohol/drogas & 16 & 15 & 28 & 15 & & & \\
\hline Trastornos esquizofrénicos & 51 & 52 & 32 & 37 & & & \\
\hline Trastornosdel humor & 6 & 12 & 12 & 18 & & & \\
\hline Trastornos ansiedad/adaptación & 5 & 8 & 20 & 20 & & & \\
\hline Trastornos de personalidad & 13 & 6 & 7 & 8 & & & \\
\hline \multicolumn{8}{|l|}{ Enfermedad (CGI) } \\
\hline Incierta & 6 & 3 & 9 & 2 & \multirow[t]{5}{*}{$\chi^{2}=28.7$} & \multirow[t]{5}{*}{15} & \multirow[t]{5}{*}{.017} \\
\hline Ligeramente & 6 & 5 & 6 & 16 & & & \\
\hline Moderadamente & 16 & 28 & 28 & 20 & & & \\
\hline Claramente & 46 & 44 & 38 & 41 & & & \\
\hline Extremadamente grave & 26 & 20 & 19 & 21 & & & \\
\hline \multicolumn{8}{|c|}{ Resultados** (estimada por el paciente) } \\
\hline Optimista & 70 & 73 & 72 & 75 & \multirow[t]{2}{*}{$\chi^{2}=.59$} & \multirow[t]{2}{*}{3} & \multirow[t]{2}{*}{ n.s. } \\
\hline Pesimista & 30 & 27 & 28 & 25 & & & \\
\hline \multicolumn{8}{|c|}{$\operatorname{Resultados}^{* *}$ (estimada por el clínico) } \\
\hline Optimista & 28 & 41 & 36 & 40 & \multirow[t]{2}{*}{$\chi^{2}=3.2$} & \multirow[t]{2}{*}{3} & \multirow{2}{*}{ n.s. } \\
\hline Pesimista & 72 & 59 & 64 & 60 & & & \\
\hline
\end{tabular}

$(*)$ Valor de $\mathrm{P}$ para diferencias entre subgrupos.

n.s. no significanción.

(**) Alta (expectativas en un año: Estado de salud física y mental).

vez, demostraron una percepción ostensiblemente más elevada que los pacientes de más edad (tabla III). El estado civil y/o el hecho de tener una relación de confianza íntima difería según los grupos (tabla II). El estado civil casado/cohabitando presentaba una elevada relación con el nivel de 'percepción moderada'(tabla II). Los pacientes viudos mostraban con significativamente mayor frecuencia una 'muy escasa percepción'. La mayoría de los pacientes con 'muy escasa percepción' no contaban con una relación de confianza íntima (tabla II). Los pacientes con esquizofrenia o trastornos orgánicos mostraron en su mayor parte una 'escasa percepción' (tabla II). Los individuos que padecían dependencias del alcohol o las drogas mostraron con significativamente mayor frecuencia una 'percepción moderada'. La probabilidad de una 'buena percepción' era más elevada entre los pacientes con trastornos del estado anímico, 
Tabla III

Asociaciones entre percepción,características demográficas, autonomía (GAF) y salud física ymental

\begin{tabular}{lccccccccc} 
& $\begin{array}{c}\text { Muy pobre } \\
\text { "insight" } \\
\mathrm{n}=67\end{array}$ & \multicolumn{2}{c}{$\begin{array}{c}\text { Pobre } \\
\text { "insight" } \\
\mathrm{n}=81\end{array}$} & $\begin{array}{c}\text { Moderado } \\
\text { "insight" } \\
\mathrm{n}=133\end{array}$ & $\begin{array}{c}\text { Bueno } \\
\text { "insight" } \\
\mathrm{n}=137\end{array}$ & $\begin{array}{c}\text { valor } \\
\text { de P* }\end{array}$ \\
\hline Variable & media & SD & media & SD & media & SD & media & SD & sign. \\
\hline Edad en el periodo de estudio & 50.1 & 19.7 & 45.8 & 16.6 & 42.2 & 12.2 & 41.8 & 13.6 & $<.001$ \\
Edad con el primer tratamiento & 40.2 & 21.2 & 37.0 & 15.9 & 32.8 & 13.2 & 32.7 & 15.6 & .004 \\
Funcionamiento global (GAF) & 46.9 & 16.0 & 49.7 & 15.9 & 52.8 & 16.7 & 49.7 & 15.9 & n.s. \\
Salud (valorada por el paciente) & 64.8 & 24.1 & 59.3 & 27.9 & 59.4 & 23.4 & 44.5 & 19.7 & $<.001$ \\
Salud (valorada por el clínico) & 50.7 & 19.8 & 50.3 & 18.0 & 54.8 & 18.3 & 54.0 & 19.6 & n.s. \\
\hline
\end{tabular}

(*) Valor de la P para diferencias enre subgrupos.

La significación fue calculada con ANOVA de una vía.

n.s. no significación.

de ansiedad o de adaptación. De los pacientes cuya gravedad de enfermedad se valoró como incierta o leve (CGI) se obtuvo significativamente con mayor frecuencia una 'percepción moderada' o una 'buena percepción'(tabla II). Una 'muy escasa percepción' se asociaba con elevados grados de autovaloración de salud física y mental (tabla III). El Funcionamiento General (GAF), (tabla III), el grado de salud física y mental establecido por el personal clínico (tabla III) y el optimismo (pesimismo) de los pacientes y el personal clínico en relación con los resultados (tabla II), no resultaron influyentes.

La distribución de los factores relacionados con el tratamiento en los cuatro niveles de percepción se presenta en la tabla IV.

El tiempo de contacto (primer contacto o contacto de larga duración relativo a determinados servicios), la observancia o cumplimiento de la medicación, la duración del tratamiento e ingresos previos, no influyeron en los niveles de la percepción del paciente. Sin embargo, los pacientes con una 'escasa percepción'presentaban significativamente con mayor frecuencia al menos un ingreso durante el estudio. Los pacientes con 'percepción moderada' o 'buena percepción' valoraban mejor su tratamiento (en términos de coste económico) si se comparaban con los pacientes con 'escasa percepción’ o 'muy escasa percepción'.

En el análisis discriminante (los datos se muestran en una tabla) se utilizaron como variables dependientes los factores que se recogen a continuación: edad, sexo, estado civil, confidente íntimo, edad durante el primer tratamiento, diagnóstico, CGI, GAF, cumplimiento de la medicación, modalidad de tratamiento (paciente externo/paciente interno), autovaloración del grado de salud, grado de salud valorado por el personal clínico, optimismo con relación a los resultados, paciente de primer contacto/paciente de larga duración, validez del tratamiento (en términos económicos), y efectos secundarios de la medicación. La percepción de la enfermedad se utilizó como variable de clase. De acuerdo con el análisis, tres coeficientes de función discriminante, a saber, la edad (Wilks' lambda ,83, p < ,001), salud física y mental autovalorada (Wilks' lambda ,89, p < ,001) y validez del tratamiento (Wilks'lambda ,79, $\mathrm{p}<, 001$ ) se diferenciaban significativamente entre los cuatro grupos de percepción. Se alcanzó un índice del 
Table IV

Asociaciones entre percepción y variables de tratamiento

\begin{tabular}{|c|c|c|c|c|c|c|c|}
\hline & $\begin{array}{l}\text { Muy pobre } \\
\text { "insight" }\end{array}$ & $\begin{array}{l}\text { Pobre } \\
\text { "insight" }\end{array}$ & $\begin{array}{l}\text { Moderado } \\
\text { "insight" }\end{array}$ & $\begin{array}{l}\text { Bueno } \\
\text { "insight" }\end{array}$ & & & \\
\hline Variable & $\begin{array}{c}\mathrm{n}=67 \\
\%\end{array}$ & $\begin{array}{c}\mathrm{n}=81 \\
\%\end{array}$ & $\begin{array}{c}\mathrm{n}=133 \\
\%\end{array}$ & $\begin{array}{c}\mathrm{n}=137 \\
\%\end{array}$ & $\chi^{2}$-Test & df & $\begin{array}{c}\text { sign. } \\
\text { Valor } \mathrm{P}^{*}\end{array}$ \\
\hline \multicolumn{8}{|c|}{ Contacto } \\
\hline Primer contacto con los pac & entes 46 & 46 & 45 & 52 & \multirow[t]{2}{*}{$\chi^{2}=1,5$} & \multirow[t]{2}{*}{3} & \multirow[t]{2}{*}{ n.s. } \\
\hline En el curso de tratamiento & 54 & 54 & 55 & 48 & & & \\
\hline \multicolumn{8}{|l|}{ Cumplimiento tratamiento } \\
\hline Cumplidor & 94 & 85 & 91 & 94 & \multirow[t]{2}{*}{$\chi^{2}=14,8$} & \multirow[t]{2}{*}{12} & \multirow[t]{2}{*}{ n.s. } \\
\hline No cumplidor & 6 & 15 & 9 & 6 & & & \\
\hline \multicolumn{8}{|l|}{ Duración tratamiento } \\
\hline$<2$ años & 39 & 39 & 30 & 34 & \multirow[t]{4}{*}{$\chi^{2}=7,6$} & \multirow[t]{4}{*}{12} & \multirow[t]{4}{*}{ n.s. } \\
\hline $2-5$ años & 16 & 16 & 22 & 22 & & & \\
\hline 6-10 años & 13 & 16 & 12 & 16 & & & \\
\hline$>10$ años & 32 & 29 & 36 & 28 & & & \\
\hline \multicolumn{8}{|l|}{ Admisiones previas } \\
\hline 0 & 30 & 32 & 34 & 36 & \multirow[t]{4}{*}{$\chi^{2}=4,1$} & \multirow[t]{4}{*}{9} & \multirow[t]{4}{*}{ n.s. } \\
\hline 1 & 16 & 15 & 16 & 17 & & & \\
\hline $2-5$ & 26 & 35 & 29 & 28 & & & \\
\hline 6 o más & 28 & 18 & 21 & 19 & & & \\
\hline \multicolumn{8}{|l|}{ Admisiones durante el estudio } \\
\hline 0 & 50 & 42 & 70 & $5 \mathrm{~B}$ & \multirow[t]{2}{*}{$\chi^{2}=10,4$} & \multirow[t]{2}{*}{3} & \multirow[t]{2}{*}{015} \\
\hline 1 o más & 50 & 58 & 30 & 42 & & & \\
\hline \multicolumn{8}{|l|}{ Valor del tratamiento } \\
\hline $0-100$ öS & 67 & 62 & 41 & 38 & \multirow[t]{3}{*}{$\chi^{2}=22,9$} & \multirow[t]{3}{*}{6} & \multirow[t]{3}{*}{$<, 001$} \\
\hline 101-500 öS & 20 & 30 & 35 & 41 & & & \\
\hline$>500 \mathrm{öS}$ & 13 & 8 & 24 & 21 & & & \\
\hline
\end{tabular}

* Valor de P para diferentes subgrupos. n.s. no significación.

44\% de clasificación correcta de grupo, en comparación con una selección natural del $25 \%$. El mayor porcentaje de asignación al grupo correcto fue el relacionado con 'buena percepción'(68\%), seguido por 'percepción moderada' $(42 \%)$ y 'escasa percepción’ (27\%). Un porcentaje de tan sólo un $15 \%$ fue la asignación al grupo 'muy escasa percepción'.

\section{Discusión}

Este estudio da como resultado la creación de un índice de desarrollo propio para la medición de la percepción de la enfermedad mental. Para el personal clínico en concreto, el índice puede resultar interesante porque es sencillo de utilizar y también muy breve. Además, la revisión de la bibliografía mas relevante demuestra que las magnitudes de índice elegidas (conciencia de estar enfermo, conocimiento de la enfermedad o los síntomas, expectativas de tratamiento, clasificación de la percepción por parte del entrevistador) componen una medida global de la percepción. Sin embargo, los hallazgos han de ser interpretados dentro de las limitaciones del estudio. Son necesarios estudios posteriores para determinar la validez y fiabilidad del índice. Uno de los mayores problemas en 
investigación es que los resultados no pueden compararse entre sí debido al enorme número de mediciones heterogéneas basadas en conceptos diferentes. Para mejorar la comparabilidad, debería desarrollarse un instrumento de consenso acerca de la percepción de la enfermedad mental.

Hallamos que la percepción se corresponde con varios factores demográficos, de historial clínico y de tratamiento. Este informe es una contribución importante a la investigación sobre la percepción de la enfermedad mental, porque llevamos a cabo el estudio sobre amplias muestras de pacientes psicóticos y no psicóticos. El nivel de escasa percepción se halló predominantemente en tres grupos diferentes: (1) en pacientes con poco contacto social, (2) en pacientes que sufren de esquizofrenia o trastornos orgánicos y (3) en pacientes que clasificaron su enfermedad física y mental como menos grave, mientras que el personal clínico la clasificara como grave.

Hasta donde nosotros conocemos, la percepción de pacientes psiquiátricos no psicóticos no ha sido estudiada hasta ahora. Sin embargo, el $12 \%$ de todos nuestros pacientes no psicóticos mostraron 'muy escasa percepción': 11 de estos pacientes no psicóticos sufrían dependencias del alcohol y las drogas, 6 padecían trastornos orgánicos y 3 sufrían de ansiedad o de trastornos de adaptación. Se encontró muy escasa percepción entre los pacientes con trastornos orgánicos, lo que es consustancial desde el punto de vista del diagnóstico. Los estudios contemplan que la escasa percepción de los pacientes esquizofrénicos está causada por su debilidad cognitiva (Fennig et al. 1996, Lysaker y Bell 1994, McEvoy et al. 1996). La carencia de capacidad cognitiva en el caso de trastornos orgánicos también apoya esta interpretación. Los pacientes con 'muy escasa percepción' o 'escasa percepción' eran significativamente de mayor edad en el momento de su primer tratamiento psiquiátrico. La influencia del diagnóstico debería tenerse en cuenta para explicar estos resultados. Los pacientes con trastornos orgánicos son a menudo de más edad en el momento de su primer tratamiento y también muestran una menor percepción. El $45 \%$ de los pacientes con esquizofrenia presentaban 'muy escasa percepción' o 'escasa percepción'. Una comparación de todos los diagnósticos demostró que una 'escasa percepción' está significativamente asociada con la esquizofrenia (véase también Fennig et al. 1996; Carpenter et al. 1973). A diferencia de Amador et al. (1994), hallamos como el diagnóstico de trastorno del estado de ánimo está relacionado con 'buena percepción'. En la mayoría de los casos no resultó posible entrevistar a los pacientes maníacos. Sin embargo, durante los episodios maníacos la percepción del paciente disminuye. Son necesarios exámenes posteriores para profundizar en los cambios en la percepción de acuerdo con las diferentes fases de la enfermedad. Los pacientes con dependencias del alcohol y/o las drogas presentaban las puntuaciones más altas de percepción. La motivación del tratamiento podría explicar este resultado. La rehabilitación de las dependencias del alcohol y/o las drogas fracasaría sin motivación para comenzar un programa de rehabilitación. Es obvio que la percepción de la enfermedad mental y la motivación para el tratamiento están asociadas. Los pacientes con dependencias tienen que ser conscientes de su enfermedad, de lo contrario el tratamiento no podría tener éxito en absoluto, es más, los pacientes no estarían en disposición siquiera de comenzar el tratamiento.

Teniendo en cuenta los ingresos, asumimos que la percepción está asociada con un resultado favorable en la fase de seguimiento. Esta idea fue verificada y el resultado está confirmado por la bibliografía (Lin et al. 
1979, McGlashan y Carpenter 1981, Heinrichs et al. 1985, Bartko et al. 1988, McEvoy et al. 1989b). A diferencia de otros (Lysaker y Bell 1994, Bartko et al. 1988, Lin et al. 1979, McEvoy et al. 1989a , 1989b), no se halló asociación entre el cumplimiento de la medicación prescrita y la percepción. Es de suponer que las respuestas por conveniencia social sean la causa de los resultados diferentes. Sólo el 8\% de nuestra muestra al azar confesó desconocer la medicación preventiva. Sin embargo, otros estudios aportan que el cumplimiento de la medicación se encuentra entre el 25\% y el 50\% (Boyd et al. 1974, Blackwell 1973, Busson y Dunn 1986, Sackett y Snow 1979).

El análisis discriminante muestra que la edad temprana, la baja autovaloración de la salud física y mental y la alta validez del tratamiento (en términos económicos) predecían una buena percepción. Sin embargo, la irregularidad del modelo discriminante es en cierto modo poco satisfactoria, sugiriendo que otras variables pueden diferenciar mejor entre grados de percepción, ya que se consiguió una clasificación correcta de grupo para tan sólo el $44 \%$ de los pacientes.

Nuestros hallazgos indican que un pequeño número de pacientes no psicóticos y también algunos pacientes con trastornos orgánicos carecían de percepción. No obstante, una pobre percepción estaba positivamente asociada con una enfermedad grave e ingresos hospitalarios durante el periodo del estudio. El conocimiento de los pacientes sobre el diagnóstico, la psicopatología y el resultado deberían mejorarse mucho mas. El estímulo, la esperanza y el conocimiento de la enfermedad combinados con la lucha contra los prejuicios negativos podrían influir sobre el tratamiento, la calidad de vida y por último pero no menos importante, sobre la percepción de su enfermedad. Esto puede abrir toda una nueva caja de herramientas a utili- zar para mejorar la vida de los pacientes con enfermedades mentales.

\section{Bibliografía}

AMADOR, X.F., STRAUSS, D.H., YALE, S.A. et al. Awareness of illness in schizophrenia. Schizophrenia Bulletin, 17: 113-132, 1991.

AMADOR, X.F., STRAUSS, D.H., YALE, S.A. et al. Assessment of insight in psychosis. American Journal of Psychiatry, 150: 873-879, 1993.

AMADOR, X.F., STRAUSS, D.H., YALE, SA. et al. Awareness of illness in schizophrenia and schizoaffective and mood disorders. Archives of General Psychiatry, 51: 826-836, 1994.

BARTKO, G., HERCZOG, I., ZADOR, G. Clinical symptomatology and drug compliance in schizophrenic patients. Acta psychiatrica Scandinavica, 77: 74-76, 1988.

BLACKWELL, B. Treatment adherence. British Jour nal of Psychiatry, 129: 513-531, 1976.

BOYD, J., COVINGTON, T., STANASZEK, W. Drug defaulting, II: analysis of compliance patterns. American Journal Hospital Pharmacy, 31: 485-491, 1974.

BUSSON, M., DUNN, A. Patients' knowledge about prescribed medicines. Pharmaceutical Journal, 236: 624626, 1986.

CARPENTER, W.T. Jr.,STRAUSS, J.S., BARTKO, J.J. Flexibel systems for the diagnosis of schizophrenia. Report from the World Health Organisation International Pilot Study of Schizophrenia. Science, 182:1275-1278,1973.

COLLINS, A.,REMINGTON, G.,COULTER,K. et al. Insight, neurocognitive function and symptom clusters in chronic schizophrenia. Schizophrenia Research, 27:37-44, 1997.

CUESTA, M., PERALTA, V. Lack of insight in schizophrenia. Schizophrenia Bulletin, 20: 359-366, 1994.

DAVID, A.S., BUCHANAN, A., REED, A. et al. The assessment of insight in psychosis. British Journal of Psy chiatry, 161: 599-602, 1992.

DAVID, A.S. On insight and psychosis: discussion paper. The Journal of the Royal Society of Medicine, 83: 325-329, 1990.

ENDICOTT, J., SPITZER, R.L., FLEISS, R.L. et al. The Global Assessment Scale:A procedure for measuring 
overall severity of psychiatric disturbance. Archives of General Psychiatry, 33: 766-771, 1976.

ESKEY, A. Insight and prognosis. Journal of Clinical Psychology, 14: 426-429, 1958.

FENNIG, S.,EVERETT, E.,BOMET, E.J. et al. Insight in first-admission psychotic patients. Schizophrenia Rese arch, 22: 257-263, 1996.

GREENFIELD, D., STRAUSS, J.S., BOWERS, M.B. et al. Insight and interpretation of illness in recovery from psychosis. Schizophrenia Bulletin, 15: 245-252, 1989.

HEINRICHS, D.W., COHEN, B.P., CARPENTER, W.T. Early insight and the management of schizophrenic decompensation. Journal of Nervous and Mental Disease, 173: 133-138, 1985.

LIN, I.F., SPIGA, R.,FORTSCH, W. Insight and adherence to medication in chronic schizophrenics. Journal of Clinical Psychiatry, 40: 430-432, 1979.

LYSAKER, P.H., BELL, M.D. Insight and cognitive impairment. Journal of Nervous and Mental Disease, 182: 656-660, 1994.

MARKOVÁ, I., BERRIOS, G. Insight in clinical psychiatry revisited. Comprehensive Psychiatry, 5: 367-376, 1995.

MARKOVÁ, I., BERRIOS, G. The assessment of insight in clinical psychiatry:a new scale. Acta Psychiatri ca Scandinavica, 86: 159-164, 1992.

McEVOY, J.P.,ALAND, J.,WILSON, W.H. et al. Measuring chronic schizophrenic patients' attitudes toward their illness and treatment. Hospital and Community Psy chiatry, 32: 856-858, 1981.

McEVOY, J.P., APPELBAUM, P.S., GELLER, L.J. et $a l$. Why must some schizophrenic patients be involuntarily committed? The role of insight. Comprehensive Psy chiatry, 10: 13-17, 1989a.

McEVOY, J.P.,APPERSON, L.J., APPELBAUM, P.S. et al. Insight in schizophrenia. Its relationship to acute schizophrenia. Journal of Nervous and Mental Disease, 177: 43-47, 1989b.

McEVOY, J.P.,FRETER, S.,MERRIT, M. et al. Insight about psychosis among outpatients with schizophrenia. Hospital and Community Psychiatry, 44: 883-884,1993.

McEVOY, J.P., HARTMAN, M.,GOTTLIEB, D. et al. Common sense, insight, and neuropsychological test per- formance in schizophrenia patients. Schizophrenia Bulle tin, 22: 635-441, 1996.

McGLASHAN, T.H.,CARPENTER, W.T. Jr. Does attitude toward psychosis relate to outcome? American Journal of Psychiatry, 138:797-801,1981.

National Institute of Mental Health. 028. CGI Clinical Global Impressions. In: GUY, W., BONATO, R.R., eds. ECDEU Assessment Manual for Psychopharmacology Rev. ed. Rockville, Maryland, 217-222, 1976.

PERALTA, V., CUESTA, M. Lack of insight: Its status within schizophrenic psychopathology. Society of Biologi cal Psychiatry 36: 559-561, 1994.

RUDAS, S. On measuring the changes in psychiatric care systems: results in an urban area (Vienna). Psychiatric Bulletin, 14: 262-266, 1990.

SACKETT, D.,SNOW, J. The magnitude of compliance and noncompliance. In:HAYNES, R., TAYLOR, D.,SACKETT. D., eds. Compliance and Health Cave. Baltimore: Journal Hopkins University Press, 1979.

SHAFFER, D.,GOULD, M.S.,BRASIC, J., et al. Children's Global Assessment Scale [CGAS]. Archives of General Psychiatry, 40: 1228-1231, 1978.

SMITH, T.E., HULL, J.W., GOODMAN, M. et al. The relative influences of symptoms, insight, and neurocognition on social adjustment in schizophrenia and schizoaffective disorder. The Journal of Nervous and Mental Disease, 187: 102-108, 1999.

SOSKIS, D.A., BOWERS, M.B. The schizophrenic experience:A follow-up study of attitude and post hospital adjustment. Journal of Nervous and Mental Disease, 149: 443-449, 1969.

TOWNSEND, J.M. Cultural conceptions and mental illness: a controlled comparison of Germany and America. Journal of Nervous and Mental Disease, 160:409-421,1975.

VAN PUTTEN, T., CRUMPTON, E., YALE, C. Drug refusal in schizophrenia and the wish to be crazy. Archives of General Psychiatry, 33: 1443-1446, 1976.

Dirección para correspondencia:

Dr. Andrea Lang

Qualitymangement / Community Mental

Health Services

Steigenteschgasse 142

A-1220 Vienna

AUSTRIA 
168 ANDREA LANG ET AL. 